\title{
MRI utility in predicting extraprostatic extension of prostate cancer and biochemical recurrence after radical prostatectomy
}

\author{
Natalia Majchrzak , Piotr Cieśliński , Tomasz Milecki², Maciej Głyda1,3, \\ Katarzyna Karmelita-Katulska ${ }^{4}$
}

\author{
${ }^{1}$ Transplantology, General Surgery and Urology Department, Poznan District Hospital, Poznan, Poland \\ ${ }^{2}$ Department of Urology and Oncological Urology, Poznan University of Medical Sciences, Poznan, Poland \\ ${ }^{3}$ Hepatobiliary and General Surgery Department, Collegium Medicum in Bydgoszcz, Nicolaus Copernicus University, Bydgoszcz, Poland \\ ${ }^{4}$ Department of General Radiology and Neuroradiology, Poznan University of Medical Sciences, Poznan, Poland
}

Introduction. The study aimed to evaluate the performance of multiparametric magnetic resonance imaging (mpMRI) and the Martini model to predict extraprostatic extension (EPE) and biochemical recurrence (BCR) of prostate cancer (PCa). Materials and methods. 61 patients underwent a radical laparoscopic prostatectomy. The preoperative risk of EPE was determined using mpMRI and the Martini model.

Results. MpMRI predicts the presence of EPE of PCa with a sensitivity and specificity of $47.4 \%$ and $85.7 \%$, respectively (AUC 0.66, 95\% Cl: 0.51-0.82, $\mathrm{p}=0.046$ ). The Martini model's sensitivity was higher, but the specificity was lower than that of mpMRI and was $84.2 \%$ and $66.7 \%$, respectively (AUC $0.78,95 \% \mathrm{Cl}: 0.66-0.89, \mathrm{p}<0.001$ ). Univariate and multivariate Cox analysis indicated that EPE in mpMRI (HR 6.6, 95\% Cl: 1.8-24.1), and the presence of positive surgical margins (PSM) (HR 7.1, 95\% Cl: 1.9-26.7) are independent factors increasing the probability of BCR.

Conclusions. MpMRI and Martini model are valuable tools in local staging of PCa, managing and predicting the oncological treatment outcomes of patients with PCa.

Key words: prostate cancer, multiparametric magnetic resonance imaging, radical prostatectomy, biochemical recurrence, extraprostatic extension

\section{Introduction}

Prostate cancer (PCa) is the most commonly diagnosed malignant neoplasm in men in the world [1]. A radical prostatectomy (RP), next to radiotherapy (RT), is the treatment of choice in patients with non-metastatic PCa [2]. Biochemical recurrence (BCR) after RP affects 30\% of patients and is one of the risk factors for disease progression and death $[3,4]$. The confirmed risk factors for the occurrence of $B C R$ in the postoperative report are: positive surgical margins (PSM) and extraprostatic extension (EPE)/locally advanced disease (stageT3-T4) [5]. The presence of the above parameters determines adjuvant treatment, i.e. local $\mathrm{RT}$, aimed at reducing the risk of disease recurrence.

Precise local staging is an essential clinical issue due to its significance in treating patients with non-metastatic PCa.

\section{How to cite:}

Majchrzak N, Cieśliński P, Milecki T, Głyda M, Karmelita-Katulska K. MRI utility in predicting extraprostatic extension of prostate cancer and biochemical recurrence after radical prostatectomy. NOWOTWORY J Oncol 2021; 71: 197-201. 
According to the current standards, the local staging is based on clinical examination (digital rectal exam - DRE) or a transrectal ultrasound (TRUS) [2]. Apart from the clinical examination and TRUS, local staging can also be performed based on mpMRI. MpMRI provides valuable information about the clinical significance and localization of the lesion [6]. This information is used to qualify the patient for a prostate biopsy. According to the current guidelines, it is recommended to perform mpMRI in suspected PCa before the first prostate biopsy [2]. Moreover, mpMRI, in the case of PCa, allows for a characterization of such clinical parameters as lesion size, possible EPE or seminal vesicle invasion (SVI) [6]. The above information allows for an estimation of the appropriate risk group of disease progression, choose the treatment method (RP vs. RT), or plan the extension of the RP. Nevertheless, mpMRI is still not validated as a diagnostic tool for local staging and treatment planning $[2,7,8]$.

The study aimed to evaluate the utility of $\mathrm{mpMRI}$ in the prediction of EPE and the impact of EPE on the occurrence of BCR in patients qualified for RP.

\section{Material and methods}

The study group consisted of 61 patients diagnosed with PCa who underwent laparoscopic RP. RP was performed either by the transor retroperitoneal approach. An extended lymphadenectomy was performed in the case of high-risk and intermediate-risk cancer with a predicted probability of lymph node involvement above 7\%, according to the Briganti 2017 model [9]. The operation was performed with bilateral or unilateral NVB preservation or without NVB preservation. The NVB preserving technique involved inter- or intra-fascial dissection of the bundles, according to Walz [10]. The procedures without NVB preservation involved a wide extra-fascial prostatectomy [10]. The decision to preserve NVB was made depending on the EAU risk group. The preoperative risk of EPE was determined according to the Martini et al. model [11]. The decision to preserve NVB was also influenced by comorbidities, erectile dysfunction present before the planned procedure, age, and the patient's preferences.

The biopsy material and specimen acquired during RP underwent histopathological assessment conducted by three pathologists in accordance with the guidelines of the International Society of Urological Pathology (ISUP) 2014 in the field of pathomorphological diagnosis of PCa [12].

The study used mpMRI obtained when qualifying the patients for the first biopsy due to suspected PCa 1.5T (GE Healthcare Medical System Optima MR360, Chicago, IL, USA) and 3T equipment (Siemens HealthCare Magnetom Skyra, Erlangen, Germany) were utilized using 12- or 18-channel Body Matrix coils. The mpMRI scheme followed the PIRADS v. 2.0 guidelines of the American College of Radiology (ACR) [6]. It included a multiplanar assessment of the prostate in T1-and T2-weighted images, diffusion weighted imaging (DWI) and a dynamic contrast-enhanced (DCE) MRI. Apparent diffusion coefficient (ADC) maps were developed automatically. The mpMRI was evaluated by four radiologists experienced in prostate imaging who knew the PSA levels and rectal examination (DRE).

Based on mpMRl, a targeted cognitive biopsy of the prostate combined with a systematic biopsy was performed, guided with transrectal ultrasound using a biplane transducer with simultaneous imaging of both planes (BK Medical Flex 400, Herlev, Denmark).

The biopsy was performed according to the scheme recommended by the European Society of Urology (EAU) [2], 6-8 specimens were collected from each lobe, plus additionally 2-4 specimens from the suspicious lesion depending on its size $[2,13,14]$. The biopsy was performed by four urologists.

BCR was diagnosed when two PSA levels above $0.2 \mathrm{ng} / \mathrm{ml}$ were obtained. PSA was monitored every three months during the first year and every six months in consecutive years.

\section{Statistical analysis}

The analyzed parameters were described using an arithmetic mean, standard deviation, and median. The normality of distribution was verified using the Shapiro-Wilk test in each of the analyzed groups. In a normal distribution, the t-Student test for independent variables was used to compare the two groups. In the case of non-normal distribution, the non-parametric Mann-Whitney test was applied. Categorical variables in individual groups were described using percentage values; they were compared using the Chi ${ }^{2}$ test with the Yates correction and with Fisher's exact test. Receiver operating characteristic (ROC) curves were determined for the analyzed parameters (Martini model and mpMRI). AUC (area under curve) was subsequently calculated, and their significance was analyzed. Cut-off points for which sensitivity and specificity reach optimal values were determined for parameters with significant AUC (Youden point).

Kaplan-Meier analysis and the log-rank test were used to compare BCR-free survival for patients with EPE in mpMRI. For BCR risk factors, hazard ratios (HRs) with 95\% confidence intervals (Cls) were determined using the Cox model. The univariate and multivariate model was established. The assumed $p$-value was $<0.05$. The IBM SPSS Statistics statistical package was used for the calculations.

\section{Results}

Table I presents the clinical characteristics of 61 patients undergoing RP ( $n=61)$. 19 patients $(n=19)$ were diagnosed with EPE $(+)$ and $42(n=42)$ without EPE $(-)$ in the final histopathological report post RP. Patients with EPE(+) differed statistically significantly from patients without EPE(-), in terms of the following clinical parameters: ISUP grade in the preoperative biopsy, maximum index lesion (IL) dimension in mpMRI, EPE diagnosis in mpMRI, ISUP Grade in the postoperative report, the incidence of PSM, and the incidence of BCR in follow-up. 
Table I. Clinical characteristics of patients. Comparison of clinical data between pEPE(-) and pEPE(+) patients

\begin{tabular}{|c|c|c|c|c|}
\hline Total n (\%) or median & $\begin{array}{l}\text { Overall } \\
(n=61)\end{array}$ & $\begin{array}{l}\text { pEPE(-) } \\
(n=42)\end{array}$ & $\begin{array}{l}\text { pEPE(t) } \\
(n=19)\end{array}$ & pEPE(+) vs. pEPE(-) p value \\
\hline age (median) & 65 & 65.7 & 63.4 & NS \\
\hline PSA (ng/ml) & 8.46 & 8.01 & 9.47 & NS \\
\hline PSAD (ng/ml/ml) & 0.24 & 0.22 & 0.29 & NS \\
\hline $\begin{array}{l}\text { DRE: } \\
\text { - normal } \\
\text { - abnormal }\end{array}$ & $\begin{array}{l}22(36.1) \\
39(63.9)\end{array}$ & $\begin{array}{l}18(42.9) \\
24(57.1)\end{array}$ & $\begin{array}{c}4(21) \\
15(79)\end{array}$ & NS \\
\hline $\begin{array}{l}\text { biopsy ISUP grade: } \\
\text { • } 1 \\
\text { - }>1\end{array}$ & $\begin{array}{l}35(57.4) \\
26(42.6)\end{array}$ & $\begin{array}{l}29(69) \\
13(31)\end{array}$ & $\begin{array}{c}6(31.6) \\
13(68.4)\end{array}$ & * \\
\hline prostate volume (ml) (median) & 38.9 & 39.32 & 37.86 & NS \\
\hline $\begin{array}{l}\text { PIRADS: } \\
\text { - } 1-3 \\
\text { - } 4-5\end{array}$ & $\begin{array}{l}21(34.4) \\
40(65.6)\end{array}$ & $\begin{array}{l}15(35.7) \\
27(64.3)\end{array}$ & $\begin{array}{c}6(31.6) \\
13(68.4)\end{array}$ & NS \\
\hline max diameter of IL in mpMRI (mm) & 13.8 & 12.26 & 17.32 & * \\
\hline $\begin{array}{l}\text { mpMRI EPE: } \\
\text { - } \operatorname{EPE}(-) \\
\text { - } \operatorname{EPE}(+)\end{array}$ & $\begin{array}{l}46(75.4) \\
15(24.6)\end{array}$ & $\begin{array}{c}36(85.7) \\
6(14.3)\end{array}$ & $\begin{array}{c}10(52.6) \\
9(47.4)\end{array}$ & * \\
\hline $\begin{array}{l}\text { mpMRI zone location: } \\
\text { - } \quad \text { PZ } \\
\text { - non-PZ }\end{array}$ & $\begin{array}{l}45(73.8) \\
16(26.2)\end{array}$ & $\begin{array}{l}29(69) \\
13(31)\end{array}$ & $\begin{array}{c}16(84.2) \\
3(15.8)\end{array}$ & NS \\
\hline $\mathrm{BCR}(+)$ & $11(18)$ & $3(7.1)$ & $8(42.1)$ & * \\
\hline $\begin{array}{l}\text { pathologic ISUP grade: } \\
\begin{array}{l}\cdot \quad 1 \\
\cdot \quad>1\end{array}\end{array}$ & $\begin{array}{l}25(41) \\
36(59)\end{array}$ & $\begin{array}{l}22(52.4) \\
20(47.6)\end{array}$ & $\begin{array}{c}3(15.8) \\
16(84.2)\end{array}$ & * \\
\hline PSM & $18(29.5)$ & $8(19)$ & $10(52.6)$ & * \\
\hline
\end{tabular}

PSA - prostate specific antigen; PSAD - PSA density; DRE - digital rectal exam; ISUP grade - 2014 International Society of Urological Pathology Grade; PIRADS - Prostate Imaging Reporting and Data System; mpMRI - multiparametric magnetic resonance imaging; EPE - extraprostatic extension ("- "absent, "+" present); pEPE - pathological extraprostatic extension ("-" absent, "+" present); PZ - peripheral zone; non-PZ - zone other than peripheral; BCR - biochemical recurrence; PSM - positive surgical margin; IL - index lesion; NS not significant; ${ }^{*}-p<0.05$

Analysis using the ROC curve showed that preoperative mpMRI might predict the presence of EPE of PCa with a sensitivity and specificity of $47.4 \%$ and $85.7 \%$, respectively (AUC 0.66, 95\% Cl: 0.51-0.82, p = 0.046). Taking into account the results of the first statistical analysis, which indicate that EPE may also be dependent on other clinical parameters, we examined the sensitivity and specificity of the Martini model, which uses the following data: PSA level, EPE status in mpMRI, ISUP Gleason grade, and the percentage of the biopsy specimen involvement. The Martini model's sensitivity was higher, but the specificity was lower than that of mpMRI and was $84.2 \%$ and $66.7 \%$, respectively (AUC $0.78,95 \%$ Cl: $0.66-0.89$, $\mathrm{p}<0.001$ ) (fig. 1).

All patients in the study were subject to follow-up. The mean follow-up was 38 months (95\% Cl: 34.0-42.5 months). Patients with $\mathrm{PEPE}(+)$ more frequently experienced $\mathrm{BCR}$ 42\% ( $n=8)$ vs. pEPE(-) 7\% ( $n=3)$ (HR 6.4, 95\% Cl: 1.7-24). Using univariate and multivariate Cox analysis, it was examined whether other clinical factors may also influence the occurrence of BCR. Previous prognostic factors influencing the patient's prognosis (PSA, ISUP grade, DRE, prostate volume, $\mathrm{SM}$ ) were analyzed, taking into account mpMRI (PIRADS of IL,

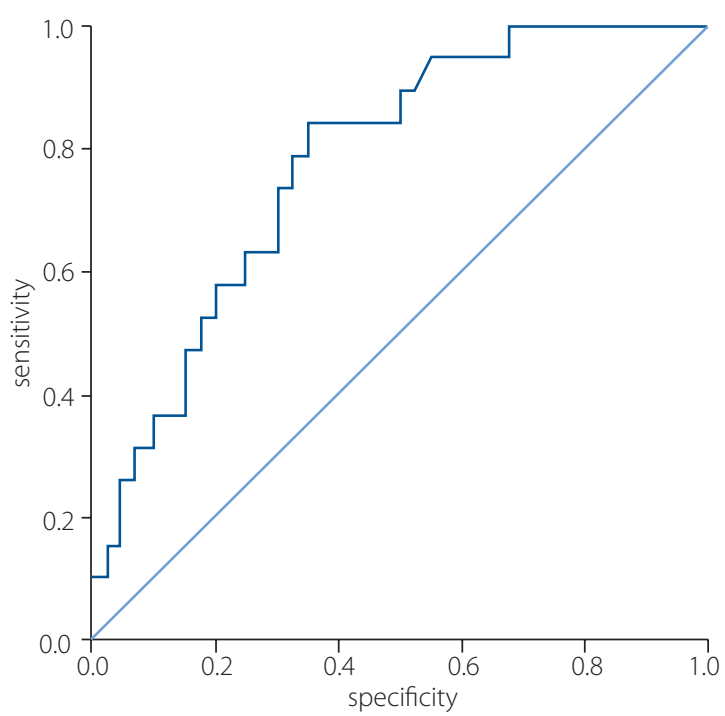

Figure 1. ROC curve - sensitivity and specificity of the Martini nomogram for EPE prediction before RP

EPE status, largest IL dimension) (tab. II). The final Cox model showed that EPE in mPMRI was an independent factor that increased the likelihood of BCR - HR 6.6 (95\% Cl: 1.8-24.1). 
Table II. Univariate and multivariate Cox regression analysis of clinical factors for BCR in patients with localized PCa after RP

\begin{tabular}{|c|c|c|c|c|}
\hline & \multicolumn{2}{|c|}{ Univariate analysis } & \multicolumn{2}{|c|}{ Multivariate analysis } \\
\hline & $\mathrm{HR}(95 \% \mathrm{Cl})$ & p value & $\mathrm{HR}(95 \% \mathrm{Cl})$ & p value \\
\hline DRE, ref. normal & $0.96(0.28-3.3)$ & 0.94 & \multirow{6}{*}{ NA } & \\
\hline PSA & $0.95(0.81-1.1)$ & 0.4 & & \\
\hline biopsy ISUP grade, ref. grade 1 & $1.76(0.56-5.8)$ & 0.35 & & \\
\hline prostate volume & $0.97(0.92-1.0)$ & 0.33 & & \\
\hline max diameter of IL in mpMRI (mm) & $1.04(0.97-1.1)$ & 0.26 & & \\
\hline PIRADS, ref. $\leq 3$ & $1.025(0.3-3.6)$ & 0.97 & & \\
\hline mpMRI - EPE, ref. EPE(-) & $3.9(1.2-12.9)$ & 0.02 & $6.6(1.8-24.1)$ & 0.005 \\
\hline SM, ref. negative & $4.7(1.4-16.1)$ & 0.01 & $7.1(1.9-26.7)$ & 0.004 \\
\hline
\end{tabular}

DRE - digital rectal exam; PSA - prostate specific antigen; ISUP grade - 2014 International Society of Urological Pathology Grade; PIRADS - Prostate Imaging Reporting and Data System; mpMRI - multiparametric magnetic resonance imaging; EPE - extraprostatic extension; BCR - biochemical recurrence; SM - surgical margins; IL - index lesion; NA - not applicable in final model; $\mathrm{Cl}$ - confidence interval; ref - reference

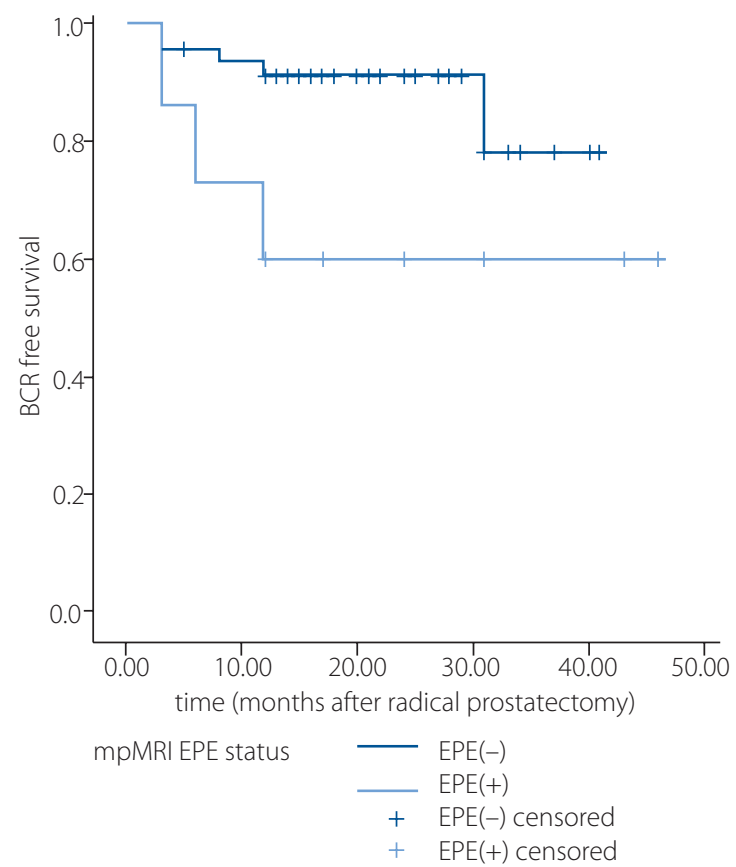

Figure 2. Kaplan-Meier survival curves for biochemical-free survival in patients with positive (+) and negative (-) mpMRI EPE

Another important factor, also significantly increasing the risk of $B C R$, was the presence of PSM in the postoperative report - HR 7.1 (95\% Cl: 1.9-26.7). Based on the above analysis, the Kaplan-Meier curve was determined for EPE assessment in mpMRI (fig. 2).

\section{Discussion}

The results obtained indicate that preoperative $\mathrm{mpMRI}$ may be a useful tool in local staging of PCa and for the prediction of recurrence; they are to a large extent consistent with the results of other similar studies $[7,8,15,16]$. The mpMRI parameters analyzed by other researchers included: PIRADS score, volume and location of index lesions, presence of EPE, and/or SVI. Moreover, taking into account mpMRI parameters in conjunction with other clinical data, such as PSA levels and prostate biopsy reports, may contribute to a significant improvement in local staging. An example of this strategy is the model according to Martini et al., which takes into account mpMRI, PSA levels, and a report of the targeted biopsy based on MRI [11].

This retrospective study is one of the few studies available in the literature, which indicates that mpMRI and the Martini model may be tools that are helpful in determining the presence of EPE before surgical treatment $[8,11]$. The sensitivity and specificity of mpMRI in assessing EPE in our study were $47.4 \%$ and $85.7 \%$, respectively, while for the Martini model $84.2 \%$ and $66.7 \%$, respectively. The meta-analysis conducted by de Rooij, assessing the diagnostic utility of mpMRI in the prediction of stage T3a (EPE), indicated a sensitivity of 0.57 (95\% Cl: 0.49-0.64) and a specificity of 0.91 (95\% Cl: 0.88-0.93) [8]. When creating this predictive model for the presence of EPE, Martini estimated the sensitivity of mpMRI alone at 40.7\%. [11]. Then, considering the mpMRI and clinical variables, the created model resulted in a higher diagnostic value than mpMRI alone (AUC for mpMRI 0.68 vs. AUC for the model 0.82) [11]. We obtained similar results in our research.

Our study also confirmed the relationship between the presence of EPE in the postoperative histopathological report and an increased risk of BCR. Moreover, we demonstrated that EPE in preoperative mpMRI might also be an essential pre-surgical factor increasing the risk of BCR after RP. Therefore, the results obtained in our study indicate that mpMRI may improve the prediction of possible disease recurrence by improving local disease staging. In a study with a similar methodology, conducted on a large group of respondents $(n=804)$, Gandalgia et al. proved that preoperative information obtained from mpMRI and the report of systematic biopsy combined with a targeted biopsy based on mpMRI allows stratification of PCa recurrence after RP [15]. When assessing the influence of preoperative factors on BCR, Manceau et al. also proved that patients with EPE(+) diagnosed in mpMRI at the treatment planning stage more frequently experience $B C R$ after RP [16]. Moreover, they showed a correlation between the occurrence of BCR and higher PIRADS scores, the greater 
maximum dimension of MRI lesions, and a higher ISUP Gleason grade in fusion biopsy [16].

From a clinical perspective, the presence of EPE in mpMRI may be a predictive factor for the risk of adjuvant RT implementation after RP. This knowledge at the treatment planning stage can help in the choice of a treatment method, i.e., RP vs. RT. If surgical treatment is chosen, the information about the presence of EPE in mpMRI and its location can be used to plan the procedure's technique and make decisions, for example, regarding the preservation of nerve bundles. It has been proven that planning RP based on mpMRI changes the operator's decision to preserve NVB in 35\% (95\% Cl: 29-41\%) of cases; this strategy is correct in $77 \%$ (95\% Cl: $72-81 \%)$ of cases and does not worsen oncological outcomes [17].

Our study has several limitations. The first significant limitation is that it was conducted on a small group studied in one center. The second limitation is the lack of assessment of the extent of EPE and its detailed location, which, according to available knowledge, may also be a significant factor influencing the risk of $B C R$.

\section{Conclusions}

MpMRI and the Martini model are helpful tools in the local staging of patients with PCa. Preoperative use of mpMRI can predict oncological treatment outcomes in patients with PCa after RP.

\section{Conflict of interest: none declared}

\section{Natalia Majchrzak}

Poznan District Hospital

Transplantology, General Surgery and Urology Department

ul. Juraszów 7-19

60-479 Poznań, Poland

e-mail:drmajchrzak@gmail.com

\section{Received: 7 Apr 2021}

Accepted: 17 Apr 2021

\section{References}

1. Ferlay J, Soerjomataram I, Dikshit R, et al. Cancer incidence and mortality worldwide: sources, methods and major patterns in GLOBOCAN 2012. Int J Cancer. 2015; 136(5): E359-E386, doi: 10.1002/ijc.29210, indexed in Pubmed: 25220842.

2. Mottet N, van den Bergh RCN, Briers E et al. European Association of Urology Guidelines. 2020 Edition. [Internet]. Vol. presented at the EAU Annual Congress Amsterdam 2020. European Association of Urology Guidelines Office; 2020. http://uroweb.org/guideline/prostate-cancer/.

3. Pound CR, Partin AW, Eisenberger MA, et al. Natural history of progression after PSA elevation following radical prostatectomy. JAMA.
1999; 281(17): 1591-1597, doi: 10.1001/jama.281.17.1591, indexed in Pubmed: 10235151.

4. Van den Broeck T, van den Bergh RCN, Arfi N, et al. Prognostic Value of Biochemical Recurrence Following Treatment with Curative Intent for Prostate Cancer: A Systematic Review. Eur Urol. 2019; 75(6): 967-987, doi: 10.1016/j.eururo.2018.10.011, indexed in Pubmed: 30342843.

5. Suardi N, Ficarra V, Willemsen P, et al. Long-term biochemical recurrence rates after robot-assisted radical prostatectomy: analysis of a single-center series of patients with a minimum follow-up of 5 years. Urology. 2012; 79(1): 133-138, doi: 10.1016/j.urology.2011.08.045, indexed in Pubmed: 22088567.

6. Barentsz JO, Choyke PL, Cornud F, et al. PI-RADS Prostate Imaging - Reporting and Data System: 2015, Version 2. Eur Urol. 2016; 69(1): 16-40, doi: 10.1016/j.eururo.2015.08.052, indexed in Pubmed: 26427566.

7. Somford DM, Hamoen EH, Fütterer JJ, et al. The predictive value of endorectal 3 Tesla multiparametric magnetic resonance imaging for extraprostatic extension in patients with low, intermediate and high risk prostate cancer. J Urol. 2013; 190(5): 1728-1734, doi: 10.1016/j. juro.2013.05.021, indexed in Pubmed: 23680307.

8. de Rooij M, Hamoen EHJ, Witjes JA, et al. Accuracy of Magnetic Resonance Imaging for Local Staging of Prostate Cancer: A Diagnostic Meta-anaIysis. Eur Urol. 2016; 70(2): 233-245, doi: 10.1016/j.eururo.2015.07.029, indexed in Pubmed: 26215604.

9. Gandaglia G, Fossati N, Zaffuto E, et al. Development and Internal Validation of a Novel Model to Identify the Candidates for Extended Pelvic Lymph Node Dissection in Prostate Cancer. Eur Urol. 2017; 72(4): 632640, doi: 10.1016/j.eururo.2017.03.049, indexed in Pubmed: 28412062.

10. Walz J, Epstein Jl, Ganzer R, et al. A Critical Analysis of the Current Knowledge of Surgical Anatomy of the Prostate Related to Optimisation of Cancer Control and Preservation of Continence and Erection in Candidates for Radical Prostatectomy: An Update. Eur Urol. 2016; 70(2): 301-311, doi: 10.1016/j.eururo.2016.01.026, indexed in Pubmed: 26850969.

11. Martini A, Gupta A, Lewis SC, et al. Development and internal validation of a side-specific, multiparametric magnetic resonance imaging-based nomogram for the prediction of extracapsular extension of prostate cancer. BJU Int. 2018; 122(6): 1025-1033, doi: 10.1111/bju.14353, indexed in Pubmed: 29676063.

12. Epstein Jl, Egevad L, Amin MB, et al. Grading Committee. The 2014 International Society of Urological Pathology (ISUP) Consensus Conference on Gleason Grading of Prostatic Carcinoma: Definition of Grading Patterns and Proposal for a New Grading System. Am J Surg Pathol. 2016; 40(2): 244-252, doi: 10.1097/PAS.0000000000000530, indexed in Pubmed: 26492179.

13. Eichler K, Hempel S, Wilby J, et al. Diagnostic value of systematic biopsy methods in the investigation of prostate cancer: a systematic review. J Urol. 2006; 175(5): 1605-1612, doi: 10.1016/S0022-5347(05)00957-2, indexed in Pubmed: 16600713.

14. Shariat, SF, Roehrborn, CG Using biopsy to detect prostate cancer. Rev Urol. 2008; 10: 262-280.

15. Gandaglia G, Ploussard G, Valerio M, et al. Prognostic Implications of Multiparametric Magnetic Resonance Imaging and Concomitant Systematic Biopsy in Predicting Biochemical Recurrence After Radical Prostatectomy in Prostate Cancer Patients Diagnosed with Magnetic Resonance Imaging-targeted Biopsy. Eur Urol Oncol. 2020; 3(6): 739-747, doi: 10.1016/j.euo.2020.07.008, indexed in Pubmed: 32847747.

16. Manceau C, Beauval JB, Lesourd M, et al. MRI Characteristics Accurately Predict Biochemical Recurrence after Radical Prostatectomy. J Clin Med. 2020; 9(12), doi: 10.3390/jcm9123841, indexed in Pubmed: 33256176.

17. Kozikowski M, Malewski W, MichalakW, et al. Clinical utility of MRI in the decision-making process before radical prostatectomy: Systematic review and meta-analysis. PLoS One. 2019; 14(1): e0210194, doi: 10.1371/ journal.pone.0210194, indexed in Pubmed: 30615661. 